\title{
Automatic Control System of Reservoir Gate
}

\author{
Yu Lin ${ }^{1, a^{*}}$, Zhiwei Lin ${ }^{2}$ and Jing $\operatorname{Tian}^{3}$ \\ ${ }^{1}$ The Department of Computer \& EEE Oxbridge College, Kunming University of Science and \\ Technology, Kunming, China \\ ${ }^{2}$ Faculty of Civil Engineering and Mechanic, Kunming University of Science and Technology, \\ Kunming, China \\ ${ }^{3}$ Yunnan Computer Software Technology Development Research Center, Kunming, China \\ akmly2011@163.com \\ * The corresponding author
}

Keywords: Automatic monitoring; Automatic control; Modbus; Forcecontrol7.0; PLC

\begin{abstract}
Reservoir is a water conservancy project of flood control and water storage, building a reservoir in the upper reaches of the river used for urban water supply, tap water and agricultural irrigation, power generation, flood control, reducing peak flow into the downstream channel through the capacity of storing flood, to achieve flood control function and multi water storage in flood season. The safety of reservoir in flood season is affected by many factors, and gate control system is accurate, timely control of the gate of the reservoir, so its reliability is a major factor affecting the safe operation of the reservoir. In this paper, combined with the actual project, automatic monitoring system of reservoir gate is put forward. This system uses the SIEMENS programmable logic controller S7-200PLC as the gate control core, through the Ethernet protocol to establish the communication connection with Forcecontrol7.0. S7-200PLC which is as master-station has two communication ports, using its own communication P0RT1 and the intelligent instruments which are as slave-station to achieve Modbus communication, monitoring the gate status, such as three-phase voltage, three-phase current, power factor to ensure the gate operation is safe and reliable.
\end{abstract}

\section{Background}

This paper takes automatic monitoring system of reservoir gate as the research object, main research objects: Forcecontrol7.0 as the core of the whole system store parameters, analyze and make intelligent judgment; S7-200 PLC is the only master-station and instruments are as slave-station in the Modbus network, between and PLC and intelligent instrument using Modbus communication; S7-200 PLC using Modbus communication protocol to acquire directly parameters from instruments such as gate height and power instrument which supports Modbus protocol to achieve real-time monitoring[1][2]. The real-time parameters of gate height power parameters, no load or load and other important parameters displays in monitoring interface. When appearing extreme operation in the gate running, the protection automatic works and prompts alarm according to the fault, processing convenient. Control of the gate is divided into "remote control" and "local control". The communication between PLC and Forcecontrol7.0 through Ethernet protocol, PLC accepts order from Forcecontrol7.0 to lift the gate remotely. Using Ethernet and Modbus protocol it can remote control reservoir gate and monitor parameters in central control room so that it can quickly respond to dangerous situations to deal with promptly to ensure the safety of the reservoir, replacing the manual observation method and improving the reservoir water gate automatic control level[3][4].

\section{System Design}

Fieldbus will be set up communication network by various kinds of intelligent instruments, to achieve remote monitoring and controlling parameter, automatic alarm, data archiving and other 
functions. Because it is an open protocol and abide by the same communication standard, users choose different manufacturers of intelligent instruments to set up communication network to exchange data according to their own requirements, improving the system versatility [5]. The operation of the equipment of the system can be timely grasp, improving the real-time performance and reliability.

Industrial Ethernet is compatible with commercial Ethernet standards in technical standards, but there are differences between them. This is mainly reflected in consider industrial demand to design commercial Ethernet at design time, in line with the requirements of the industrial field, real-time higher, high real-time performance, the time of fault network less than $300 \mathrm{~ms}$, to avoid a standstill [6]. Adopting strong anti-interference of the communication cable improves the reliability of data transmission.

This system chooses industrial Ethernet and Modbus protocol to realize the communication between the central control room and monitoring instrument. PLC and instrumentation are both using Modbus Protocol. The instruments which support the Modbus protocol survey data such as gate height, load, power factor, three-phase voltage and other parameters.

Reservoir gate is the last line of defense to ensure the safety of reservoir flood operation and downstream people's property, so operation of reservoir gate control system must be accurate and reliable. Because reservoir environment is the high humidity and the low temperature, in this case to ensure the two-way communication between PLC and Forcecontrol7.0, PLC and intelligent instruments being safe, stable, real-time and the point above is very important. Industrial Ethernet protocol and Modbus protocol come from industrial applications, so they ensure the reliability of data in the process of two-way transmission. Using the above communication can improve the automatic and intelligence level of reservoir gate control, and improve the reliability and safe operation of reservoir in flood season [7]. The Forcecontrol7.0 could monitoring the running state of reservoir gate, setting up the model to set the alarm type, the limit value and the alarm level, analysis data. If it happens such as the lack of power factor high, three-phase voltage equal, the system automatic operates shutdown protection, preventing the gate rushed top or bottom seat and automatic alarm, avoiding the occurrence of motor burning, the broken wire rope, Forcecontrol7.0 issues an alert sound to inform on duty staff and manager[8]. The working diagram of the system is shown in Fig. 1.

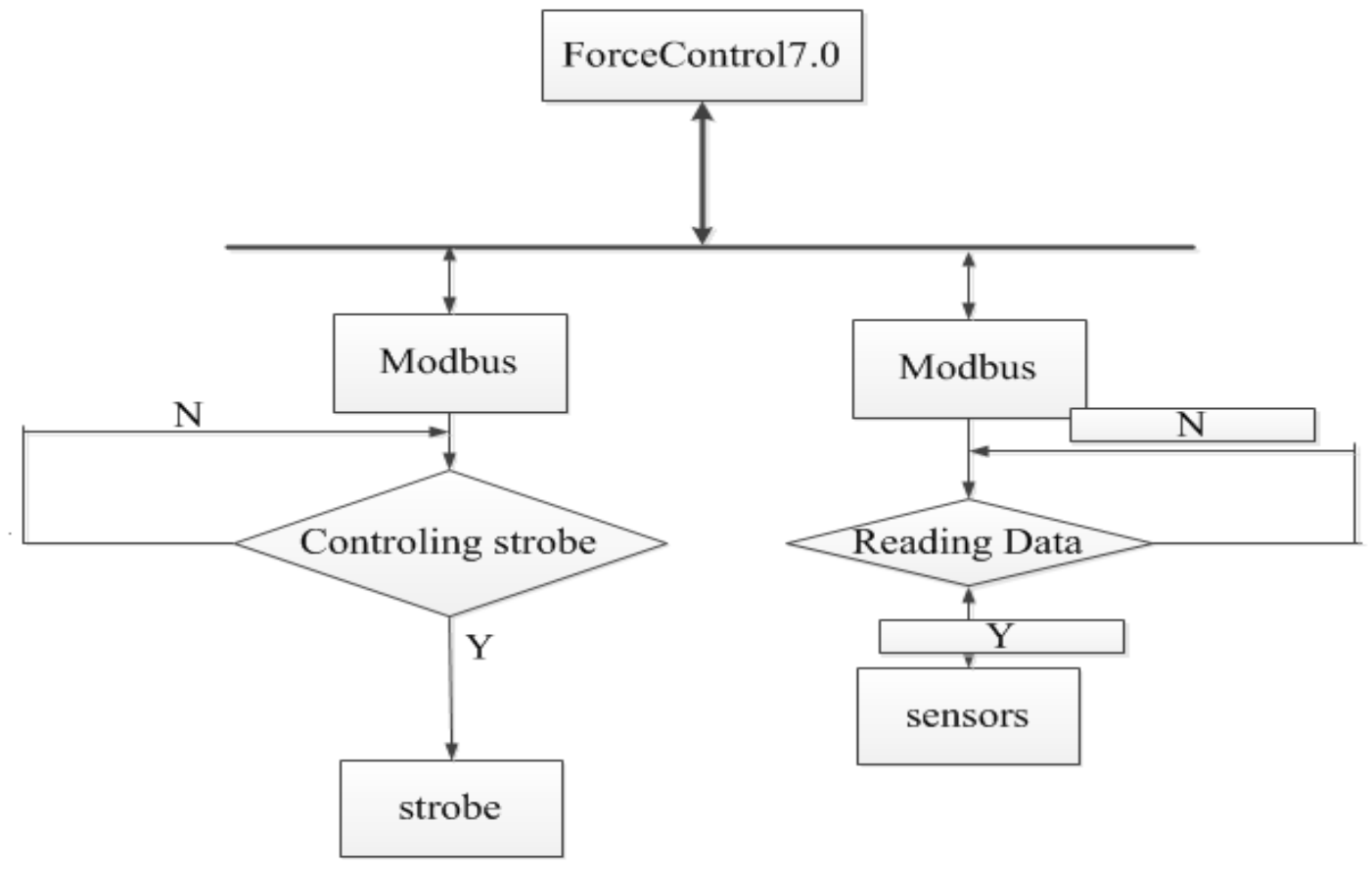

Figure 1. Working diagram of the system 


\section{System Software Design}

Gate bears the reservoir water, flood control, agricultural irrigation and other tasks, its working state directly affect the safety of the dam and downstream people's lives and property safety. Compared with the traditional gate control system, this system has a high degree of digitization and intelligence, real-time data acquisition, transmission accuracy, precise control, perfect protection mechanism to avoid the occurrence of severe safety accidents such as motor burning.

The intelligent instruments needs to be setup the slave-station address, one slave-station having only one address, setting slave-station address of instruments, reading or writing, reading or writing the number of bytes and store the starting address in the PLC internal memory where the data starts to store. PLC as master is shown

Forcecontrol7.0 through Ethernet protocol to establish data exchange channel with PLC which using Modbus communication reads real-time data from intelligent instruments to monitor the status of the gate, finally realizing the precise control of the gate. If the fault occurs, it could automatic start shutdown protection, such as stop machine protection, prevent occurrence of the secondary accidents. DTU GSM receives the alarm signal sent by Forcecontrol7.0, and sends text message to manger. Procedures mainly achieve the "intelligent control gate" and "Forcecontrol7.0 intelligent analyzing data" and make a decision whether start to alarm. In order to realize remote monitoring, gate control contains two kinds of" remote "and" in situ ". Forcecontrol7.0 makes intelligent analysis and diagnosis of data which reads from a variety of industrial instrumentation, if result exceeds a safe value, Forcecontrol7.0 automatically has shutdown protection, sends alarm sound and sends alarm message to the staff[9][10].

Forcecontrol7.0 supports SIEMENS Ethernet protocol, so it can be extended by CP243-1 to communicate with S7-200PLC.Forcecontrol7.0 through the CP243-1 can read or write all memory of S7-200PLC, so it can change PLC internal variables to achieve the remote control of the gate .For the first time CP243-1 needs to be distributed IP address, after the completion needing to

repower CP243-1, the IP address can be used effectively.

S7-200PLC supports Modbus RTU master / slave protocol, via the communication port 1 to achieve Modbus communication between the equipment which are as slave-station, such as power instrument, angle encoder, pressure sensor, water level sensor, through the external Ethernet module can and ForceControl7.0 to realize the Ethernet communication.

\section{System Operation and Test}

In the remote control mode, the manager in central control room can set gate height in the monitoring interface, clicking "up", "down", "stop" three buttons to lift the gate in place to achieve the accurate remote control of the gate depending on the Ethernet communication between Forcecontrol7.0 and PLC.

System in the process of operation, to achieve automatic and intelligent goal, it must implement actual data acquisition and processing of abnormal situation. Manager can click "control button" to control the gate remote; The system in the process of operation, to achieve the automation and intelligent processing, it must implement the accurate data acquisition and abnormal situation, to monitor the operation of the motor by power instrument when the gate running. If measured value exceeds the upper and lower bounds of the alarm, Forcecontrol7.0 providing corresponding alarm information, alarm information can be displayed in the monitoring interface to facilitate the user to understand the current status and causes of failure, PLC will send out the stop signal control, to achieve the protection of the equipment, the gate will lock until the elimination of anomalies. The test is divided into two parts: 1 . automatic monitoring system of gate operation test; 2 . meeting the constraint conditions of the alarm. The gate monitoring is shown in Fig. 2. 


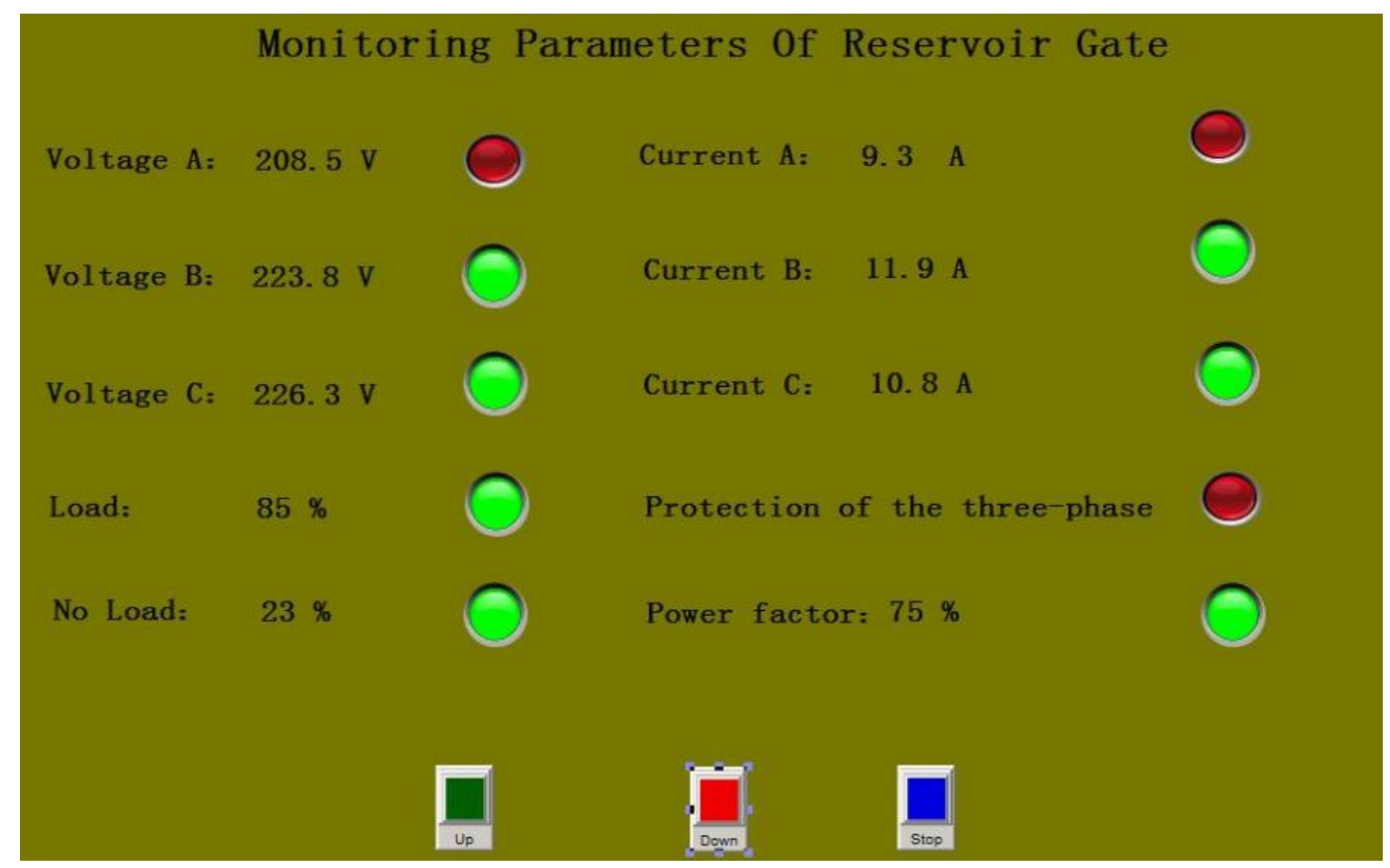

Figure 2. Monitoring interface

\section{Conclusion}

In this paper, automatic monitoring system of reservoir gate is as the object, combined the development of information technology, more detailed description of the system design process and the key issues to resolve. The system improves the intelligent control of gate, Let managers more intuitive grasp of the running state of the gate, to provide safety guarantee for control flood in flood season.

\section{References}

[1] G.H. Lou, H. Zhang, W. Zhao. Research on Designing Method of CAN BUS and Modbus Protocol Conversion Interface.2009 International Conference on Future BioMedical Information Engineering, 2009:180-182.

[2] Derwin Suhartono. Developing Controller Area Network Management Application Based on Modbus in Multi Generator Set Controller through Local Network and Internet [J]. Procedia Engineering, 50(2012): 426-435.

[3] Maria Auxiliadora Muanis Persechini.Centralized and Distributed Control Architectures under Foundation Fieldbus Network [J]. ISA Transactions, 52(2013):149-161.

[4] Xin Wang .Water Monitoring System Based on ZigBee and GPRS Original Research Article [J]. Procedia Engineering, 15(2011):2680-2684.

[5] Ping Ai. China Water Conservancy Information Assessment Study and Practice [M]. Changjiang Press, 2011:1-5.

[6] Niv Goldenberg, Avishai Wool. Accurate Modeling of Modbus/TCP for Intrusion Detection in SCADA Systems [J].International Journal of Critical Infrastructure Protection, 6(2013): 63-75.

[7] Baimin Zhang. Application of Computer Monitoring system of Sluice Gate in the south of Kong [J]. Hydropower Automation and Dam Monitoring, 34(2010):82-84.

[8] Jiang Dan. Safety design of automatic control system for reservoir gate [J]. China Water Transport, 7(2014):168-171. 
[9] Liu Cao. The Monitoring System with Redundancy for Reservoir Gate Based on AB ControlLogix [J]. mechanicaland electronical engineering, 7(2009):89-93.

[10] Jian Ni. Application of MB series PLC in automatic control system of sluice gate of Shandong River [J]. Hydropower Automation and Dam Monitoring, 34(2010):74-76. 